\title{
ACKNOWLEDGEMENT TO REFEREES
}

(C) Springer International Publishing Switzerland 2013

\section{Dear Reader}

As we reach the final issue of Molecular Diagnosis \& Therapy for 2013, we hope that you have found the articles published throughout the year to be interesting and informative. The editor and publishing staff have appreciated the high quality of content contributed to the journal this year. The publishing schedule for 2014 is well under way, and we are looking forward to bringing you many high-quality and authoritative articles over the coming year.

2013 saw the successful integration of the Adis portfolio of journals into the Springer production systems and custombuilt platforms (SpringerLink, Springer for R\&D, and Springer for Hospitals \& Health). We are confident that our authors and subscribers benefit from improved discoverability, mobile optimisation and robust delivery of content on these platforms; and Springer production capacity has allowed us to increase the number of articles published per issue whilst considerably reducing submission-to-publication times across the portfolio.

Looking ahead to 2014, the Adis portfolio will continue to grow the amount of content published while maintaining high standards. The number of Adis titles will also increase as existing quality Springer journals are brought under the Adis brand-Advances in Therapy, Targeted Oncology, and the European Journal of Drug Metabolism and Pharmacokinetics. Adis are also committed to fostering Open Access publication: all Adis titles offer the Springer Open Choice option and we are adding a further ten fully Open Access titles in specific therapy areas such as Diabetes Therapy, Cardiology and Therapy and Dermatology and Therapy, previously published under the Springer Healthcare imprint. Information about the portfolio can be found on Springer.com/Adis.

We would like to thank all the authors who have contributed articles to Molecular Diagnosis \& Therapy in the last 12 months. They have generously set aside time in their busy schedules to prepare content, and without their hard work and diligence we would not have been able to publish the journal. The quality of published articles also reflects the significant time and effort dedicated by the peer reviewers, who ensure that we continue to publish content of the highest possible standard. In addition to the members of our Honorary Editorial Board, we would like to thank the following individuals who acted as referees for articles in Molecular Diagnosis \& Therapy in 2013:

Chandrasekharan Adithan, India

Simon-Gruita Alexandra, Romania

Vanessa Almeida, Brazil

Antonis Antoniou, UK

Alicia Arnott, Australia

Babak Asghari, Islamic Republic of Iran

Stelios Assimakopoulos, Greece

Wendy Bailey, USA

Shashi Bala, USA

Linnea Baudhuin, USA

Joanna Bialek, Germany

P. S. Bisen, India

Anne Blaschke, USA

Michael A. Bookman, USA

Simona Bota, Romania

Lodewijk Brosens, The Netherlands
Stephanie Broyles, USA

Maximilian Burger, Germany

John Carlquist, USA

Dipshikha Chakravortty, India

Kishore B. Challagundla, USA

Zhong Chen, China

Louis Chesler, UK

Chien-Shun Chiou, Taiwan, Republic of China

Ewa Chmielowska, Poland

Nam Hoon Cho, Republic of Korea

Tom Chu, USA

Gisele Colleoni, Brazil

Jean-Christophe Corvol, France

James Dear, UK

Stijn Deborggraeve, Belgium

Florence Doucet-Populaire, France 
Sebastiaan Engelborghs, Belgium

Diana English, USA

Mauro Feola, Italy

Fabiana Filigheddu, Italy

Carola Forster, Germany

Pim French, The Netherlands

Eitan Friedman, Israel

Bogdan Geavlete, Romania

Felice Giangaspero, Italy

Michael Goggins, USA

Maria F. Gonzales-Escribano, Spain

Angela Gradilone, Italy

Jim W. Grey, UK

Rajani Kanth Gudipatti, USA

Medine Idrizoglu Gulcebi, Turkey

Batoul Sadat Haerian, Malaysia

H. Berner Hammer, Norway

Aaron Holley, USA

Kathryn Holt, Australia

Benjamin Horne, USA

Marcus Horstmann, Germany

Xuwei Hou, China

Marianne Samir Makboul Issac, Egypt

Tsutomu Kageyama, Japan

Kouji Kajinami, Japan

Min-Jong Kang, USA

Theocharis Karaolides, UK

W. Kassouf, Canada

Amy Keating, USA

Kiarri Kershaw, USA

Hynda Kleinman, USA

Roger Kockelbergh, UK

Slava Korshunov, USA

Marianne Kristiansen, Norway

Richard Kwon, USA

$T$.T. Lao, Hong Kong

Lu Lechun, China

Yumin Li, China

Dong Liang, USA

Zhao Limei, China

Thomas Lion, Austria

Zhanfang Ma, China

Fabrizio Maggi, Italy

Susanne Magnusson, Sweden

Johannes Mair, Austria

Anke-Hilse Maitland-van der Zee, The Netherlands

David Malkin, Canada

Nicasio Mancini, Italy

Ay Ly Margaret, Indonesia

Robert C.G. Martin, USA

Kunihiko Matsui, Japan

Manabu Memoto, Japan

Antonella Mencacci, Italy
Balraj Mittal, India

Richard G. Moore, USA

Daniel J. Mueller, Canada

James M. Mullin, USA

Anjana Munshi, India

Simona Ognjanovic, USA

Harry Ostrer, USA

Tiago Fleming Outeiro, Germany

Noel Pabalan, Canada

Milena de Paiva-Cavalcanti, Brazil

Filipe Paulo, Portugal

Kitty Pavlakis, Greece

David S. Perlin, USA

Robert Perneczky, Germany

Todd Peterson, USA

Paul Pinksy, USA

Nicholas C. Popescu, USA

Anjan Purkayastha, USA

Benito J. Reguerio, Spain

Stephen S. Roberts, USA

Anne Roivainen, Finland

Taofik Rusdiana, Indonesia

Luis A. Salazar, Chile

Linoj Samuel, USA

P. Sanz-Cameno, Spain

Beata Sarecka-Hujar, Poland

Christian Schwentner, Germany

Ladislav Senolt, Czech Republic

Theodoros N. Sergentanis, Greece

Joginder K. Sharma, India

Xinggui Shen, USA

Jane Skov, Denmark

Melissa Southey, Australia

Padma Srikanth, India

Ewa Stepien, Poland

Brian Tait, Australia

Jasmine Talameh, USA

Chong Gee Teo, USA

Hans Tillmann, Germany

Brian Tomlinson, Hong Kong

Sean van Diepen, USA

Claire F. Verschraegen, USA

Daniel Von Hoff, USA

Raj Vuppalanchi, USA

Nathan R. Wall, USA

D. Wang, USA

Kai Wang, USA

James Wilson, USA

Petra F. Wolffs, The Netherlands

Xiaojing $X u$, USA

Xiao $X u$, USA

Rutao Yao, USA

Ulrich M. Zanger, Germany 
Li-rong Zhang, China

Hong Zhang, USA
Xiaolei Zhang, USA

Wang Zuoguang, China

Along with our schedule of invited articles, we also encourage the submission of high-quality reviews and original research articles. We would like to remind to you keep Molecular Diagnosis \& Therapy in mind when deciding where to submit your research. We also welcome comment from our readers on any of our articles.

We look forward to your continued support of the journal in 2014 and to bringing you first-class content from around the globe. With best wishes from the staff of Molecular Diagnosis \& Therapy and all at Adis Publications. 